\title{
Alternatively activated M2 macrophages increase in early stages of experimental autoimmune myocarditis in Lewis rats
}

\author{
Hanseul Oh ${ }^{1, \dagger}$, Meejung Ahn ${ }^{2, \dagger}$, Yoh Matsumoto ${ }^{3}$, Taekyun Shin ${ }^{1, *}$ \\ ${ }^{1}$ Laboratory of Veterinary Anatomy, College of Veterinary Medicine and Veterinary Medical Research Institute, and \\ ${ }^{2}$ School of Medicine, Jeju National University, Jeju 690-756, Korea \\ ${ }^{3}$ Department of Immunotherapy Development, Tokyo Metropolitan Institute of Medical Science, Tokyo 156-8506, Japan
}

(Received: May 16, 2013; Revised: September 13, 2013; Accepted: October 16, 2013)

\begin{abstract}
To better understand the role of macrophages in early stages of experimental autoimmune myocarditis (EAM), we compared the expression of inducible nitric oxide synthase (iNOS) and arginase-1, markers for classically activated M1 and alternatively activated M2 macrophages, respectively, in the hearts of EAM-affected and control rats. Immunohistochemical evidence revealed that both iNOS-positive and arginase 1-positive macrophages were found in EAM lesions, while some cells were co-localized with both markers. This finding suggests that the increased level of arginase-1, which is partly from M2 macrophages, contributes to the modulation of EAM, possibly through the reduction of nitric oxide in the lesion.
\end{abstract}

Keywords : arginase-1, experimental autoimmune myocarditis, inducible nitric oxide synthase, macrophage

\section{Introduction}

Experimental autoimmune myocarditis (EAM), an autoimmune inflammatory cardiac disorder, is an animal model for human giant cell myocarditis [11, 12, 26]. Although the etiologies of giant cell myocarditis and dilated cardiomyopathy are not known, an autoimmune mechanism targeting cardiac myosin has been proposed; thus, EAM is an appropriate animal model for this human cardiac dysfunction [26]. EAM is initiated by autoreactive $\mathrm{T}$ cells and macrophages $[11,25]$. The condition is characterized by a gradual decrease in inflammatory cells through apoptosis $[9,21,27]$ followed by fibrosis in the damaged cardiac tissues leading to cardiac dysfunction [28]. The role of $\mathrm{T}$ cells in the development of EAM has been investigated [7, 16]; however, little is known about the involvement of macrophage phenotypes.

Research during the past decade has focused on the immunopathological changes in EAM lesions [26]. These changes are characterized by $\mathrm{T}$ cell and macrophage infiltration in early stages and subsequent secretion of pro-inflammatory molecules, including inducible nitric oxide synthase (iNOS) [25] and osteopontin [23] and fibrotic tissue replacing inflammatory cells, causing cardiac dysfunction in the later stages of EAM [13]. Various macrophage phenotypes play important roles in the initiation and remission of autoimmune diseases, including the experimental autoimmune encephalomyelitis (EAE) rat model $[2,24]$, particularly in the remission of inflammation during the peak stage of EAE, suggesting that a similar macrophage phenotype is involved in EAM.

Macrophages are highly versatile because local environmental factors shape their phenotypic and functional properties [14]. Macrophages are classified as classical M1 (iNOS positive) and alternatively activated M2 (iNOS negative) macrophages and vary according to their induction factors, cytokine production, and phagocytosis type $[14,15,19]$. Immunologically, cytokines reciprocally regulate iNOS and arginase activity in macrophages [17]. M2 macrophages are readily identified because they contain an abundance of arginase $[8,10]$. The induction of arginase- 1 prevents macrophages from producing nitric oxide (NO), rendering them unable to kill intracellular pathogens [22]. In addition to their immunological roles, alternatively activated macrophages may be involved in tissue remodeling because they produce several extracellular matrix proteins [6]. However, it is not known whether alternatively activated macrophages are involved in the progression of EAM.

To better understand the involvement of macrophages in the development of EAM, the present study used immunohistochemistry to detect M1 (iNOS-positive) and M2 (arginase 1-positive) macrophages in cardiac tissue during the acute phase of EAM at 2 weeks post-immunization.

*Corresponding author

Tel: +82-64-754-3363, Fax: +82-64-756-3354

E-mail: shint@jejunu.ac.kr

The first two authors contributed equally to this work. 


\section{Materials and Methods}

\section{Experimental animals}

The subjects were 6- to 8-week-old male Lewis rats purchased from Orient Bio (Korea). The rats were housed in plastic cages and maintained at $23 \pm 2^{\circ} \mathrm{C}$ under a $12: 12 \mathrm{~h}$ light-dark cycle and fed 5L79 rat formula (PMI Nutrition, USA). Food and water were provided ad libitum. All experimental procedures were conducted in accordance with the Guidelines for the Care and Use of Laboratory Animals at Jeju National University.

\section{Purification of cardiac myosin}

Cardiac myosin was prepared from the ventricular muscle of human hearts according Murakami et al. [18]. The antigen preparation consisted primarily of a $205 \mathrm{kDa}$ protein, and although it contained small amounts of several substances other than cardiac myosin, this crude antigen preparation has been used to induce EAM in rats $[1,23,25]$.

\section{Induction of experimental autoimmune myocarditis}

The cardiac myosin fraction was dissolved in phosphatebuffered saline (PBS) containing $0.3 \mathrm{M} \mathrm{KCl}$ at a concentration of $2 \mathrm{mg} / \mathrm{mL}$. The antigen solution was mixed with an equal volume of complete Freund's adjuvant (CFA) supplemented with Mycobacterium tuberculosis H37RA (Difco, USA) at a concentration of $5 \mathrm{mg} / \mathrm{mL}$. The rats were immunized with $0.1 \mathrm{mg}$ cardiac myosin in CFA subcutaneously administered to each hind footpad followed by intraperitoneal administration of $5 \mu \mathrm{g}$ of pertussis toxin (Sigma-Aldrich, USA). Age- and sex- matched Lewis rats served as controls and were challenged with PBS in CFA followed by intraperitoneal administration of $5 \mu \mathrm{g}$ pertussis toxin.

Based on the results of our previous studies [1, 23, 25], the optimal time to investigate the acutely inflamed cardiac tissue was 2 weeks post-immunization (PI). Thus, the EAMaffected rats were sacrificed under ether anesthesia on day 14 PI and the phenotype of the cardiac cells expressing iNOS, arginase 1, or both were compared between the EAM and control rats using immunohistochemistry.

\section{Antibodies}

Mouse monoclonal or rabbit polyclonal anti-arginase-1 was obtained from Santa Cruz Biotechnology (USA). Rabbit polyclonal anti-TGF- $\beta 1$ (Santa Cruz Biotechnology) for immunohistochemical staining. Rabbit polyclonal anti-iNOS was obtained from BD Biosciences (USA). Mouse monoclonal anti-rat macrophage (ED1; AbD Serotec, UK) antibody was used to identify activated macrophages.

\section{Histopathology}

Transverse sections taken through the middle of the heart specimens were sliced into two equal portions and fixed in $10 \%$ formalin for microscopic grading. The paraffin-embedded tissue was cut and stained with hematoxylin-eosin for histological examination.

\section{Immunohistochemical analysis}

Five-micrometer-thick sections of paraffin-embedded tissue were deparaffinized, heated in citrate buffer $(0.01 \mathrm{M}, \mathrm{pH}$ 6.0) in a microwave for $3 \mathrm{~min}$ and then treated with $0.3 \%$ hydrogen peroxide in methyl alcohol for 20 min to block endogenous peroxidase activity. After three washes in PBS, the sections were incubated with $10 \%$ normal goat serum (Vector ABC Elite Kit; Vector Laboratories, USA) and then incubated for $1 \mathrm{~h}$ at room temperature with primary antibody: mouse anti-arginase 1 (dilution $1: 400$ ), rabbit antiiNOS (dilution $1: 200$ ) and rabbit anti-TGF- $\beta 1$ (dilution 1 : 200). Primary antibody was omitted as a negative control. After three washes in PBS, the sections were treated with the appropriate secondary antibody (dilution $1: 200$; Vector Laboratories) for $45 \mathrm{~min}$, washed three times in PBS, and then incubated for $45 \mathrm{~min}$ with avidin-biotin peroxidase complex (Vector ABC Elite kit; Vector Laboratories), and prepared according to the manufacturer's instructions. The peroxidase was reacted using a diaminobenzidine substrate kit (Vector Laboratories). The sections were counterstained with hematoxylin, dehydrated, and cleared with xylene before being mounted on slides. The immunostained sections were further analysed in three different sections from three different animals. Microscopy images $(\times 20$ magnification, progres $\mathrm{C} 7$; Jenoptik, Germany) were automatically treated with Image J software to measure each immunoreactive intensity and the percentage (\%) of positive areas relative to the total area was calculated.

\section{Double immunofluorescence}

Double-labeling to detect the two antigens was performed as previously reported [14]. Briefly, paraffin sections were incubated with $10 \%$ normal horse serum for $1 \mathrm{~h}$, followed by the first primary antibody including mouse monoclonal antiarginase-1 $(1: 100)$, ED1 (mouse monoclonal anti-rat macrophages, $1: 200$ in dilution), and biotinylated anti-mouse $\operatorname{IgG}(1: 200$; Vector Laboratories) for $1 \mathrm{~h}$ at room temperature. After washing, the sections were incubated with tetramethylrhodamine isothiocyanate-labeled streptavidin $(1: 500$, Zymed; Invirogen, USA) for $1 \mathrm{~h}$ at room temperature. Then the sections were washed and incubated with second primary antibodies $(1: 100$ rabbit polyclonal anti-arginase 1,1 : 100 rabbit polyclonal anti-iNOS, or $1: 100$ rabbit polyclonal anti-TGF- $\beta 1)$ followed by biotinylated anti-rabbit $\operatorname{IgG}(1$ : 200; Vector Laboratories) overnight at $4^{\circ} \mathrm{C}$. After washing, the sections were incubated with tetramethylrhodamine isothiocyanate-labeled streptavidin (1:500, Zymed; Invitrogen) for $1 \mathrm{~h}$ at room temperature. For counterstaining, 0.001 $\mu \mathrm{g} / \mathrm{mL}$ 4',6-diamidine-2-phenylindole, dihydrochloride (DAPI; Thermo Fisher Scientific, USA) in PBS was added and incubated at room temperature for $20 \mathrm{~min}$. The immunofluorescence-stained specimens were examined under a fluorescence microscope (BX-51; Olympus, Japan). After photographing, 

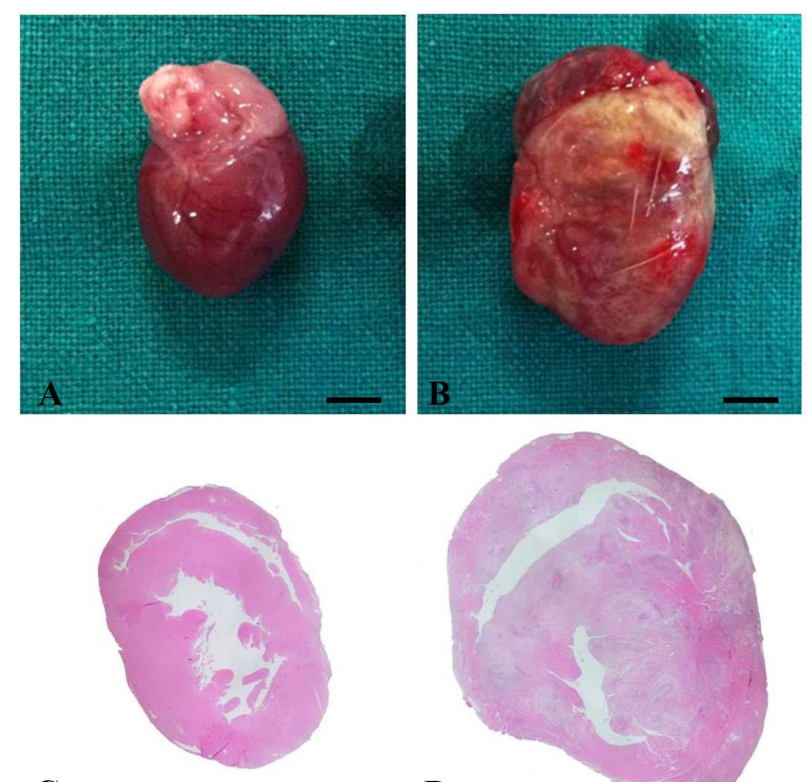

C
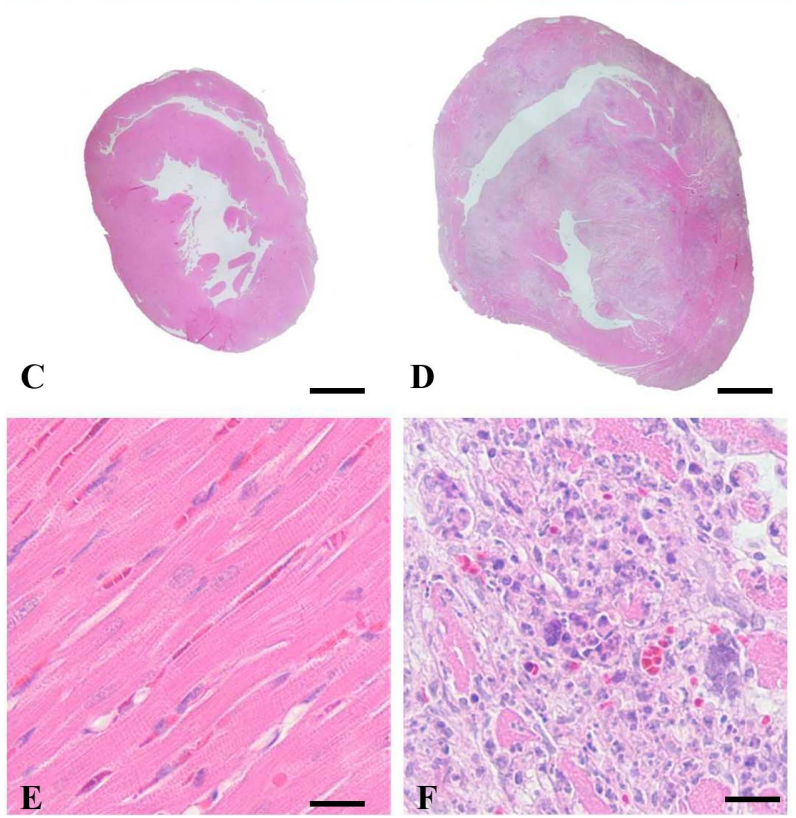

Fig. 1. Gross (A and B) and histopathological findings (C-F) of the hearts with CFA-immunized controls (A, C and $\mathrm{E})$ and the hearts with EAM (B, D and F). The upper panels (A and B) exhibit the gross findings and both the middle and lower panels (C-F) show the histological changes in hematoxylin and eosin stained the heart tissue. Scale bars $=5 \mathrm{~mm}$ (A and B), $2 \mathrm{~mm}(\mathrm{C}$ and D), $50 \mu \mathrm{m}$ (E and F).

the images were merged to visualize colocalization using Adobe Photoshop 7.0 software (Adobe Systems, USA).

\section{Statistical analysis}

Data are expressed as mean $\pm \mathrm{SE}$. The data from two groups were analyzed by Student $\mathrm{t}$ test. The $p$ value $<0.05$ was considered significant.

\section{Results}

\section{Gross and histopathological changes}

Fig. 1 shows representative examples of the histopathological findings in CFA-immunized control (Figs. 1A, C, and E) and EAM hearts on day 14 PI (Figs. 1B, D, and F). Gross findings included marked enlargement of the EAM heart and spotty changes in color on the surface (Fig. 1B) compared to that of the CFA-immunized control (Fig. 1A). The change in color was the result of inflammatory cell infiltration and myocardial necrosis. Cross-sections of the heart revealed that the pericardium and ventricular myocardium of the EAMaffected heart (Fig. 1C) were thicker and paler than those of the control heart (Fig. 1D). Furthermore, massive infiltration of macrophages and $\mathrm{T}$ cells was observed in the pericardium and ventricular myocardium of the EAM-affected hearts (Fig. $1 \mathrm{~F})$, as shown in previous studies $[3,23]$.

\section{Immunohistochemical analysis}

No arginase 1-positive immunoreactivity was found in any area of the CFA-immunized control hearts (Fig. 2A). On day $14 \mathrm{PI}$, inflammatory cells in the EAM-affected heart showed intense immunostaining for arginase 1 (Fig. 2D). No iNOS immunostaining was found in the heart of CFA-immunized rats (Fig. 2B); however, several inflammatory cells in the EAM lesions were positive for iNOS (Fig. 2E).

Strong TGF- $\beta 1$ immunoreactivity was found only in the endothelial cells of the blood vessels in the CFA-immunized control hearts (Fig. 2C); whereas damaged cardiocytes in the myocardium of the EAM hearts were positive for TGF- $\beta 1$ on day 14 PI. Moreover, weak TGF- $\beta 1$ immunostaining was detected in a few infiltrating inflammatory cells in the EAM lesions (Fig. 2F). Semiquantitative analysis of immunoreactions using Image $\mathrm{J}$ has shown that the percentage area of arginase 1 immunoreactivty in the EAM lesions $(16.1 \pm$ $2.26 \%)$ was significantly higher $(p<0.01)$ compared with CFA-immunized control $(2.5 \pm 0.02 \%$ ) (Fig. $2 \mathrm{G}$ ), and that a similar pattern of increased dimension was also recognized in iNOS expression in the EAM lesions $(10.1 \pm 0.43 \%)$ compared with CFA-immunized control $(0.3 \pm 0.04 \%)(p<$ 0.001 ) (Fig. $2 \mathrm{H}$ ). The significant upregulation of TGF- $\beta 1$ was also recognized in the EAM lesions $(14.9 \pm 2.21 \%)(p<$ $0.05)$ compared with CFA-immunized control $(8.3 \pm 1.19 \%)$ (Fig. 2I).

\section{Macrophage phenotype}

To verify the phenotype of the macrophages that infiltrated EAM-affected hearts, double immunofluorescence staining with ED1 was performed using arginase 1 or iNOS. Arginase 1 immunoreactivity was detected in ED1-positive macrophages (Figs. 3A-D). Similarly, iNOS immunoreactivity was detected in a few ED1-positive macrophages (Figs. 3E-H). Moreover, arginase 1 was occasionally immunopositive in iNOS-positive macrophages in EAM lesions (Figs. $3 \mathrm{I}-\mathrm{L}$ ), indicating that the M1 and M2 markers were colocalized in some cells. Furthermore, TGF- $\beta 1$ immunoreactivity was colocalized with arginase 1-positive macrophages (Figs. 3M-P).

\section{Discussion}

Our study is the first to find classically and alternatively activated macrophages in early inflammatory lesions in hearts affected by EAM. The distinct early appearance of M2 

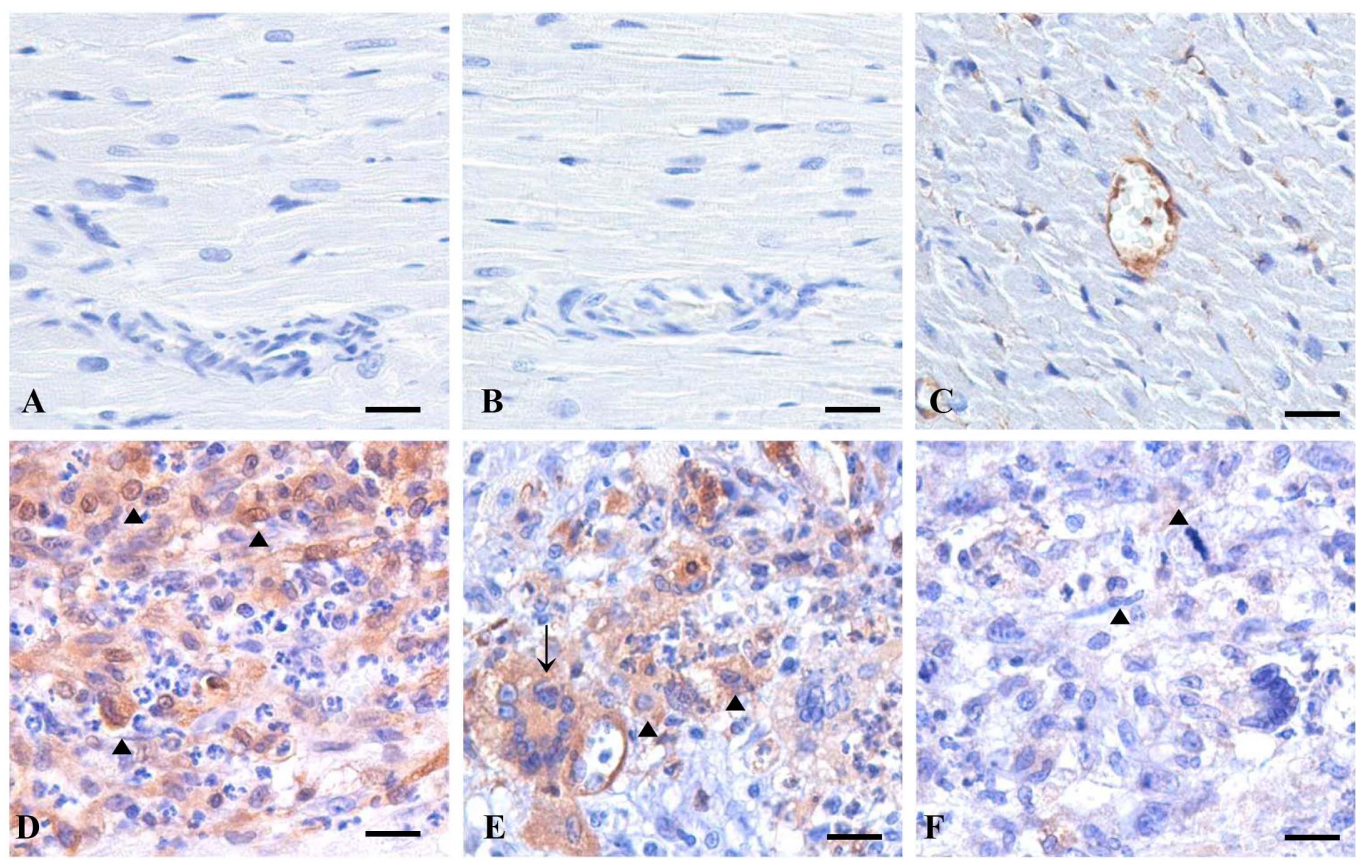

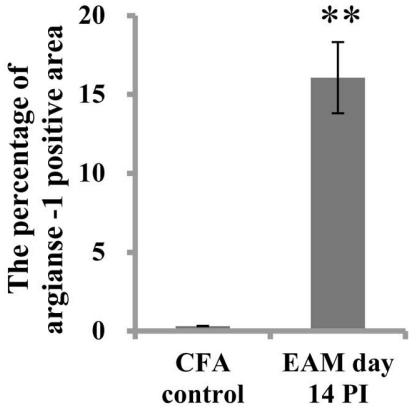

G

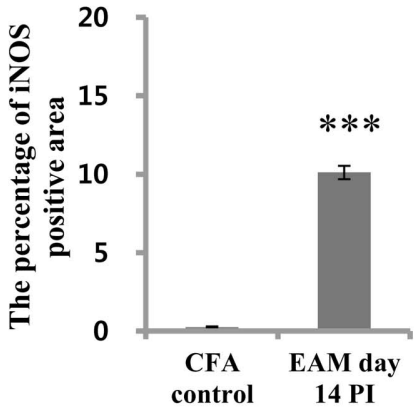

H

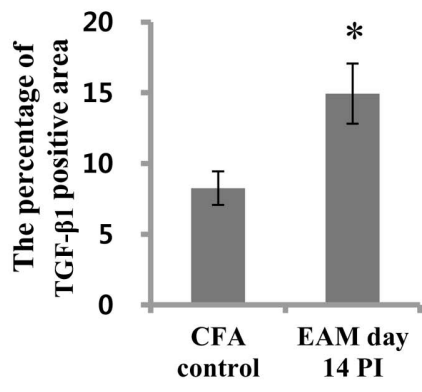

I

Fig. 2. Immunohistochemical analysis of arginase $1(A, D)$, iNOS $(B, E)$ and TGF- $\beta 1(C, F)$ in the rat hearts with CFA-immunized control (A, B and C) and with EAM on day 14 PI (D, E and F). The bar graphs (G, H and I) shows the percentage of immunopositive areas of each arginase 1 , iNOS and TGF- $\beta 1$ in immunostained sections using Image $J$ software. The percentage of immunopositive areas of each arginase $1(\mathrm{G})$, iNOS $(\mathrm{H})$, and TGF- $\beta 1$ (I) was significantly increased in the EAM affected hearts compared with those of CFA control, respectively. The data in bar graphs (G, H and I) are presented as mean \pm SE. ${ }^{*} p<0.05,{ }^{*} p<0.01, * * * p<0.001 v s$. CFA-immunized controls. H\&E. Scale bars $=25 \mu \mathrm{m}(\mathrm{A}-\mathrm{F})$.

macrophages suggests that this phenotype plays an important role in the remission of inflammation in EAM-affected hearts.

The results of our immunofluorescence double staining experiment revealed macrophages that were positive for either iNOS or arginase 1 and some that were positive for both, suggesting the existence of three macrophage phenotypes in early stages of EAM: iNOS-positive M1, arginase 1positive M2, and iNOS- and arginase 1-positive M1/M2, macrophages.

EAM is characterized by the loss of cardiomyocytes and infiltration of autoimmune $\mathrm{T}$ cells and macrophages in early stages of inflammation, followed by replacement with fibrosis in the later stage and cardiac dilatation $[4,26,28]$. To explain the immunomodulatory factors involved in the suppression of pro-inflammatory T cells and M1 macrophages in EAM, we hypothesize that the anti-inflammatory mediators of M2 macrophages compete with pro-inflammatory factors in the early inflammatory EAM lesions.

The fibrosis observed in the EAM hearts may be explained by the association between $\mathrm{M} 2$ macrophages and the production of several extracellular matrix proteins [6] and TGF- $\beta 1$ in the present study. It was also postulated that Th2 cytokines including TGF- $\beta 1$, markedly enhanced in the maximum inflammatory phase of EAM, modulate the inflammation in the course of EAM in rat models [20]. Considering that TGF- $\beta 1$ signaling plays a critical role in promoting alternative macrophage activation [5], the macrophages expressing 

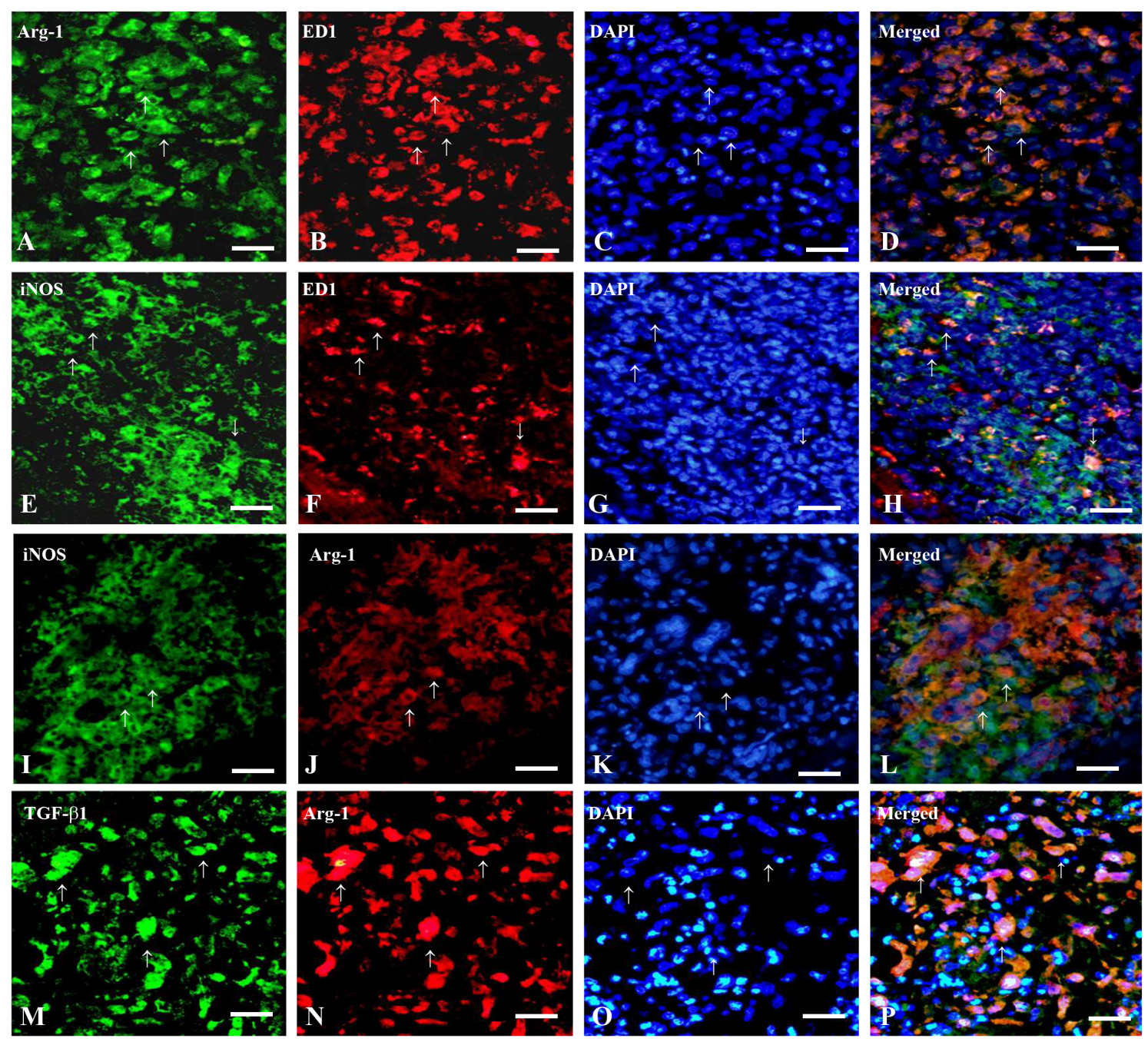

Fig. 3. Double immunofluorescence staining of arginase 1 with ED1 (A-D), iNOS with ED1 (E-H), iNOS with arginase 1 (I-L) and TGF- $\beta 1$ with arginase 1 (M-P) in the hearts affected with EAM on day 14PI. (A-D) Arrows indicate arginase 1 (A, green) positive macrophages colocalized with ED1 (B, red) in the EAM lesions (D, merged). (E-H) A few iNOS (E, green) positive macrophages were colocalized with ED1 (F, red) in the EAM lesions (H, merged; arrows). (I-L) iNOS (I, green) was immunopositive for arginase 1 ( $\mathrm{J}$, red) in the same macrophages in the EAM lesions (L, merged; arrows). (M-P) TGF- $\beta 1$ immunoreactivity (M, green) was colocalized with arginase $1(\mathrm{~N}$, red) in the EAM lesions (P, merged; arrows). The sections were counterstained with DAPI (C, G, K, O). Scale bars $=50 \mu \mathrm{m}(\mathrm{A}-\mathrm{P})$.

TGF- $\beta 1$ in our study may have been involved in the activation of M2 macrophages. The mechanisms underlying cardiac fibrosis and dilatation of the heart in the later stage of EAM are complex and warrant further study.

Collectively, these findings suggest that M2 macrophages play an important role in the progression of EAM, and that the application and/or preferential induction of M2 macrophages is an alternative therapy for cardiac autoimmune diseases.

\section{References}

1. Ahn M, Kim H, Matsumoto Y, Shin T. Increased expression of caveolin-1 and -2 in the hearts of Lewis rats with experimental autoimmune myocarditis. Autoimmunity 2006, 39, 489-495.

2. Ahn M, Yang W, Kim H, Jin J, Moon C, Shin T. Immunohistochemical study of arginase-1 in the spinal cords of Lewis rats with experimental autoimmune encephalomyelitis. Brain Res 2012, 1453, 77-86.

3. Blyszczuk P, Berthonneche C, Behnke S, Glönkler M, Moch H, Pedrazzini T, Lüscher TF, Eriksson U, Kania G. Nitric oxide synthase 2 is required for conversion of pro-fibrogenic inflammatory $\mathrm{CD}_{133^{+}}$progenitors into $\mathrm{F} 4 / 80^{+}$ macrophages in experimental autoimmune myocarditis. Cardiovasc Res 2013, 97, 219-229.

4. Cihakova D, Rose NR. Pathogenesis of myocarditis and dilated cardiomyopathy. Adv Immunol 2008, 99, 95-114.

5. Gong D, Shi W, Yi S, Chen H, Groffen J, Heisterkamp 
N. TGF $\beta$ signaling plays a critical role in promoting alternative macrophage activation. BMC Immunol 2012, 13, 31.

6. Gratchev A, Guillot P, Hakiy N, Politz O, Orfanos CE, Schledzewski K, Goerdt S. Alternatively activated macrophages differentially express fibronectin and its splice variants and the extracellular matrix protein betaIG-H3. Scand J Immunol 2001, 53, 386-392.

7. Hanawa $\mathbf{H}$, Inomata $\mathbf{T}$, Okura $\mathbf{Y}$, Hirono $\mathbf{S}$, Ogawa $\mathbf{Y}$, Izumi T, Kodama M, Aizawa Y. T cells with similar Tcell receptor $\beta$-chain complementarity-determining region 3 motifs infiltrate inflammatory lesions of synthetic peptides inducing rat autoimmune myocarditis. Circ Res 1998, 83, 133-140.

8. Ho VWH, Sly LM. Derivation and characterization of murine alternatively activated (M2) macrophages. Methods Mol Biol 2009, 531, 173-185.

9. Ishiyama S, Hiroe M, Nishikawa T, Shimojo T, Abe S, Fujisaki H, Ito H, Yamakawa K, Kobayashi N, Kasajima T, Marumo F. The Fas/Fas ligand system is involved in the pathogenesis of autoimmune myocarditis in rats. J Immunol 1998, 161, 4695-4701.

10. Johann AM, Barra V, Kuhn AM, Weigert A, von Knethen A, Brüne B. Apoptotic cells induce arginase II in macrophages, thereby attenuating NO production. FASEB J 2007, 21, 2704-2712.

11. Kodama M, Matsumoto Y, Fujiwara M. In vivo lymphocyte-mediated myocardial injuries demonstrated by adoptive transfer of experimental autoimmune myocarditis. Circulation 1992, 85, 1918-1926.

12. Kodama M, Matsumoto Y, Fujiwara M, Masani F, Izumi T, Shibata A. A novel experimental model of giant cell myocarditis induced in rats by immunization with cardiac myosin fraction. Clin Immunol Immunopathol 1990, 57, 250-262.

13. Kodama M, Matsumoto Y, Fujiwara M, Zhang S, Hanawa H, Itoh E, Tsuda T, Izumi T, Shibata A. Characteristics of giant cells and factors related to the formation of giant cells in myocarditis. Circ Res 1991, 69, 1042-1050.

14. Martinez FO, Helming L, Gordon S. Alternative activation of macrophages: an immunologic functional perspective. Annu Rev Immunol 2009, 27, 451-483.

15. Martinez FO, Sica A, Mantovani A, Locati M. Macrophage activation and polarization. Front Biosci 2008, 13, 453-461.

16. Matsumoto Y. New approach to immunotherapy against organ-specific autoimmune diseases with $\mathrm{T}$ cell receptor and chemokine receptor DNA vaccines. Curr Drug Targets Immune Endocr Metabol Disord 2005, 5, 73-77.
17. Mori M, Gotoh T. Arginine metabolic enzymes, nitric oxide and infection. J Nutr 2004, 134, 2820S-2825S.

18. Murakami U, Uchida K, Hiratsuka T. Cardiac myosin from pig heart ventricle. Purification and enzymatic properties. J Biochem 1976, 80, 611-619.

19. Neumann H, Kotter MR, Franklin RJM. Debris clearance by microglia: an essential link between degeneration and regeneration. Brain 2009, 132, 288-295.

20. Okura Y, Yamamoto T, Goto S, Inomata T, Hirono S, Hanawa H, Feng L, Wilson CB, Kihara I, Izumi T, Shibata A, Aizawa Y, Seki S, Abo T. Characterization of cytokine and iNOS mRNA expression in situ during the course of experimental autoimmune myocarditis in rats. $\mathrm{J}$ Mol Cell Cardiol 1997, 29, 491-502.

21. Ratcliffe NR, Hutchins J, Barry B, Hickey WF. Chronic myocarditis induced by $\mathrm{T}$ cells reactive to a single cardiac myosin peptide: persistent inflammation, cardiac dilatation, myocardial scarring and continuous myocyte apoptosis. J Autoimmun 2000, 15, 359-367.

22. Rutschman R, Lang R, Hesse M, Ihle JN, Wynn TA, Murray PJ. Cutting edge: Stat6-dependent substrate depletion regulates nitric oxide production. J Immunol 2001, 166, 2173-2177.

23. Shin T, Ahn M, Kim H, Kim HM, Matsumoto Y. Increased expression of osteopontin in the heart tissue of Lewis rats with experimental autoimmune myocarditis. J Vet Med Sci 2006, 68, 379-382.

24. Shin T, Ahn M, Matsumoto Y. Mechanism of experimental autoimmune encephalomyelitis in Lewis rats: recent insights from macrophages. Anat Cell Biol 2012, 45, 141-148.

25. Shin T, Tanuma N, Kim S, Jin J, Moon C, Kim K, Kohyama K, Matsumoto Y, Hyun B. An inhibitor of inducible nitric oxide synthase ameliorates experimental autoimmune myocarditis in Lewis rats. J Neuroimmunol 1998, 92, 133-138.

26. Suzuki J, Ogawa M, Watanabe R, Morishita R, Hirata Y, Nagai R, Isobe M. Autoimmune giant cell myocarditis clinical characteristics, experimental models and future treatments. Expert Opin Ther Targets 2011, 15, 1163-1172.

27. Suzuki K, Izumi T, Iwanaga T, Fujita T, Shibata A. Multinucleated giant cells undergoing apoptosis in experimental autoimmune myocarditis. Arch Histol Cytol 1995, 58, 231-241.

28. Watanabe K, Sukumaran V, Veeraveedu PT, Thandavarayan RA, Gurusamy N, Ma M, Arozal W, Sari FR, Lakshmanan AP, Arumugam S, Soetikno V, Rajavel V, Suzuki K. Regulation of inflammation and myocardial fibrosis in experimental autoimmune myocarditis. Inflamm Allergy Drug Targets 2011, 10, 218-225. 